\title{
A Heuristic-Based Appliance Scheduling Scheme for Smart Homes
}

\author{
Anish Jindal, Bharat Singh Bhambhu, Mukesh Singh, Neeraj Kumar, and Kshirasagar Naik
}

\begin{abstract}
The ever-growing demand for electricity in the residential sector results in creating a severe burden on electric grids. However, with the emergence of smart homes (SHs) and smart grids (SGs), this burden can be reduced to some extent. To address this issue, we propose an energy management system in this paper which manages the power requirements of SHs automatically according to the utility constraints and user priorities. The proposed system is based on a heuristic technique which considers the user's priority and power available from the grid as well as distributed energy resources (DERs) for scheduling of appliances. It works by dividing the appliance scheduling problem in an SH into sub-problems for different time-slots. Then, a heuristic solution is designed for each sub-problem. The instantaneous load demands are handled in real-time to comply with the available power from the grid/utility. The data from different SHs is gathered to test the performance of the proposed scheme in real-time. Results show that the proposed scheme efficiently manages the load demand of the SH with respect to power available from the utility, battery energy storage system, and user preferences.
\end{abstract}

Index Terms-Appliance scheduling, battery energy storage system, energy management system, heuristic approach, smart home.

\section{INTRODUCTION}

With an exponential increase in energy consumption of the residential sector, the load demand of consumers is exceeding the existing capabilities of conventional power resources such as-coal, natural gas, and oil. Therefore, the burden on conventional grids is escalating, which creates the risk of grid breakdown. To mitigate this issue and to create a robust system, it is necessary to design an electrical grid which takes intelligent decisions to distribute its power. This can be achieved by using smart grids (SGs), which integrate information and communication technology (ICT) within the existing grid infrastructures [1].

SG increases the efficiency and sustainability of the existing power system networks. In SG, real-time power demands of industrial, commercial, and residential sectors are managed in such a manner that the gap between the demand and

A. Jindal is with the School of Computing \& Communications, Lancaster University, UK (e-mail: anishjinda190@gmail.com).

B. S. Bhambhu is with the Delta Electronics India Private limited, Gurgaon, India (e-mail: bs12bhambhu@gmail.com).

M. Singh is with the Electrical \& Instrumentation Engineering \& Engineering Department, Thapar Institute of Engineering \& Technology, Patiala (Punjab), India (e-mail: mukesh.singh @ thapar.edu).

N. Kumar is with the Computer Science \& Engineering Department, Thapar Institute of Engineering \& Technology, Patiala (Punjab), India (e-mail: neeraj.kumar@thapar.edu).

K. Naik is with the Electrical and Computer Engineering Department, University of Waterloo, Canada (e-mail: snaik@uwaterloo.ca) the generation can be reduced. Managing the load of the residential sector plays a crucial role in reducing this gap to maintain the grid stability [2]. However, this is a challenging task as the residential sector is complex and have multiple appliances operating at different instants of time. To achieve this task, the appliances in homes need to be scheduled based on the power supplied by the utility. Moreover, maximizing the use of distributed energy resources (DERs) and battery energy storage system (BESS) in homes helps in addressing this problem. Hence, we can design an intelligent appliance scheduling scheme in smart homes ( $\mathrm{SHs}$ ) to reduce the demand and supply gap [3].

Appliances in SHs can be controlled with the help of ICT [4]. These appliances are connected to a local SH controller via home area network (HAN), using technologies such as IEEE 802.15.4, ZigBee, and Wi-Fi [5]. This controller schedules the appliances intelligently to perform day-to-day operations automatically in order to enhance the user's comfort. Several research proposals have been reported in the literature for this purpose. For instance, Li and Jayaweera [6] developed a framework for resource pooling amongst the SHs. The authors proposed an hierarchical architecture for maintaining grid stability in which the extra power available with SHs was channelized to the microgrids. Zenginis et al. [7] presented a cooperative stragtegy for the buildings to reduce the cabon emmisions and operating cost of the microgrids by energy exchange between the buildings using a common DC bus.

Managing the demand of the residential sector in an effective way also helps to reduce the peak load on the grid. For instance, Chang et al. [8] proposed the architecture of a co-ordinated energy management system for minimizing the cost of balancing the supply and demand in a neighborhood. Authors modeled the deferrable loads as a Markov decision process problem and then used dynamic programming to solve individual energy management problems for minimizing the power balancing cost. Wang et al. [9] used the concept of shared energy storage for managing the load in households. The energy storage resources were shared between end users and network operators which helped in reducing the overall energy cost of the users while managing their demand response. Tushar et al. [10] proposed an auction-based control mechanism for such shared storage facilities in the residential sector in order to manage the demand response. Jindal et al. [11] proposed a scheme to manage the load of homes in order to reduce the peak load demand on grid by analyzing the consumption data of various appliances in a house. However, none of the aforementioned schemes considered users' preferences and usage of DERs in SHs while designing 
the demand response mechanisms.

To address this issue, many researchers have incorporated the concept of DERs while formulating the demand response management strategies. DERs can be used as the primary or secondary source of power for managing the partial load demand of SHs. These DERs are generally solar photovoltaic (PV) panels, battery storage systems, fuel cells, and plug-in hybrid vehicles that can provide power to the $\mathrm{SH}$ as and when required [12]. DERs help in reducing the burden on SG during peak hours so that the grid stability is maintained. To maximize their utilization in the household energy management, Tushar et al. [13] proposed a scheme in which the energy generated from solar PV was shared amongst households and grid. Authors also minimized the overall cost of operating such a shared facility by introducing the concept of virtual cost. Although the DERs have been considered in the literature while designing energy management systems, but their main objective was to minimize the cost [14]-[16]. For instance, Bui et al. [17] proposed a hierarchical energy management system for coordinating the energy management for the buildings by using the adjustable power to save the operating cost of the network. In an another study, Althaher et al. [18] considered dynamic electric pricing for managing the load requirements. Their aim was to minimize the cost while providing comfort to the users. The branch and bound technique was used by researchers for scheduling of the devices by considering realtime pricing information and customer's constraints [19]. For example, Chavali et al. [20] designed a distributed framework to minimize the cost while scheduling the devices in an $\mathrm{SH}$ using a greedy technique. The major difference between the existing proposals [8], [9], [14]-[16], [18]-[20] and the proposed scheme is that the focus of earlier proposals was on cost minimization for the users rather than on creating a power balance between power demand and supply in the smart grid. Therefore, these proposals overlook the utility conditions while scheduling the loads in SHs, which can result in a power imbalance at the grid. Moreover, [8], [9] have not studied the impact of DERs in the overall demand management process. Though the DERs and shared ownership of energy storage have been incorporated in a few of the proposals [7], [10], [13], [14], [17], but these have not taken user preferences and comfort into account. All these factors are considered in the proposed scheme.

There are few proposals having objective which closely matches with the objective of our proposed scheme. For example, in [21], authors proposed a distributed control strategy to allocate power to each building's energy management control unit in a region. The appliances in buildings were then scheduled by solving the MILP problem such that the power consumed by each control unit is equal to the allocated power. However, the authors in [21] do not consider the DERs and the use of BESS in their proposed scheme. Moreover, their appliance admission control strategy was applicable only to a limited set of appliances having operational flexibilities with respect to their working duration. The authors in [22] presented a greedy approach based SH energy management system to schedule different appliances in an SH for cost saving and to reduce the annoyance level for the users. This approach is different from our proposed approach in a number of ways. Firstly, the authors have generalized the users' preference on the basis of a survey, whereas we have taken user preferences in each SH. Secondly, the authors used k-means clustering algorithm to make clusters of user preference profiles and assigned one profile to each $\mathrm{SH}$ for load management; whereas our scheme manages the load demand in each $\mathrm{SH}$ in a distributed manner via individual $\mathrm{SH}$ energy management system. The comparative analysis of a few aforementioned techniques with the proposed scheme in terms of technique used, use of DERs, type of load, nature, and objective of the scheme is summarized in Table I.

\section{A. Motivation}

From the discussion above, it can be concluded that the existing proposals are lacking in many aspects with respect to load management in SHs. Firstly, the existing home energy management systems largely ignore the utility conditions while scheduling the appliances. The power generation capacity of the utility is often limited, so this aspect should be considered while managing the load demand. Secondly, the users' preferences should also be taken into account while generating the appliance schedule and managing their load requirements. Thirdly, the use of BESS in SHs has not been explored to its full potential. Although the BESS has been used by the researchers in SHs, its use is often limited to store the power generated by DERs. In the proposed scheme, the energy of BESS is utilized in such a way that it acts as source (during peak hours) as well as load (during off-peak hours). The proposed scheme breaks the appliance scheduling problem into smaller sub-problems, solved using a heuristic approach, to generate the near-optimal schedule of appliances. The proposed scheme is distributed in nature (i.e., it works for individual SHs), thus it has less computational complexity, which makes it fast and reliable. Moreover, additional benefits like discounts on electricity bills and various incentives can be provided to the consumers to increase their willingness to participate in the proposed scheme.

\section{B. Contributions}

The major contributions of this work are summarized as follows.

1) An energy management system for an $\mathrm{SH}$ is proposed which manages its load demand according to the power supplied by the SG and power generated by DERs. This scheme schedules the appliances in an SH according to the utility conditions, user preferences, and considers the end times of the associated appliances so as to finish the task on time while maximizing the user comfort.

2) A heuristic-based appliance scheduling scheme is formulated to find an initial appliance schedule in an $\mathrm{SH}$. This scheme splits the scheduling problem into various sub-problems and finds an near-optimal solution for each sub-problem using the theoretical bounds on the subproblem.

3) The instantaneous changes in the load demand are also managed to maintain the balance between demand and 
TABLE I: Comparative analysis of the proposed scheme.

\begin{tabular}{|c|c|c|c|c|c|c|}
\hline S. No. & Schemes & Technique used & $\begin{array}{l}\text { Use of } \\
\text { DERs }\end{array}$ & Nature & Type of load & Objective of scheme \\
\hline 1 & [7] & MILP & Yes & Distributed & Buildings & $\begin{array}{l}\text { Reduce the carbon emmisions and cost of operating } \\
\text { the microgrid by energy exchange between } \\
\text { buildings }\end{array}$ \\
\hline 2 & [8] & Markov decision process & No & Distributed & Buildings & Minimize overall cost of the retailer \\
\hline 3 & [10] & $\begin{array}{l}\text { Vickrey auction and } \\
\text { Stackelberg game }\end{array}$ & Yes & Centralized & Residential units & $\begin{array}{l}\text { Allocation of energy from residential units to share } \\
\text { with the storage facility }\end{array}$ \\
\hline 4 & [11] & Data analytics & No & Centralized & Smart homes & $\begin{array}{l}\text { Manage the demand response in smart homes to } \\
\text { reduce the peak load in the smart grid }\end{array}$ \\
\hline 5 & [13] & $\begin{array}{l}\text { Theory of maxima and } \\
\text { minima }\end{array}$ & Yes & Centralized & Smart community & $\begin{array}{l}\text { Reduction in operational cost of shared facility } \\
\text { controller }\end{array}$ \\
\hline 6 & [14] & $\begin{array}{l}\text { Linear programming, } \\
\text { stochastic } \\
\text { scheduling }\end{array}$ & Yes & Distributed & Smart homes & Reduction in overall cost for the consumer \\
\hline 7 & [16] & MILP & Yes & Distributed & Smart homes & $\begin{array}{l}\text { Minimize the total cost of electricity usage in a } \\
\text { household }\end{array}$ \\
\hline 8 & [17] & $\begin{array}{l}\text { Hierarchical energy } \\
\text { management system }\end{array}$ & Yes & Centralized & Buildings & $\begin{array}{l}\text { Energy trading between microgrids by the use of } \\
\text { adjustable power to utilize the energy network } \\
\text { resources efficiently }\end{array}$ \\
\hline 9 & [19] & $\begin{array}{l}\text { Branch and bound } \\
\text { technique }\end{array}$ & No & Distributed & Smart homes & $\begin{array}{l}\text { Smart scheduling of heating and air-conditioning } \\
\text { devices to save energy costs }\end{array}$ \\
\hline 10 & [21] & $\begin{array}{l}\text { Average consensus } \\
\text { algorithm, MILP }\end{array}$ & No & Distributed & Buildings & $\begin{array}{l}\text { Schedule appliances according to allocated power } \\
\text { to the controllers }\end{array}$ \\
\hline 11 & [22] & Greedy approach & Yes & Centralized & Smart homes & $\begin{array}{l}\text { Save the electricity cost and to maximize the use } \\
\text { of energy generated by DER in the neighborhood }\end{array}$ \\
\hline 12 & Proposed & Heuristic approach & Yes & Distributed & Smart homes & $\begin{array}{l}\text { Manage load requirements in homes according } \\
\text { to the utility conditions so as to create a balance } \\
\text { between demand and supply }\end{array}$ \\
\hline
\end{tabular}

supply in real-time. For this purpose, appliances in an SH are shifted to different time-slots on the basis of user specified priority of the appliances and their specified end times.

\section{Organization}

The rest of the paper is organized as follows. Section II presents the energy management system of SH. Section III gives the detailed description of proposed heuristic algorithms used for scheduling the appliances. The simulation results and discussion are presented in Section IV. Finally, the paper is concluded in Section V.

\section{ENERGY MANAGEMENT IN SH}

In this paper, the appliances in an $\mathrm{SH}$ are scheduled based on the power allocated by the utility. For this purpose, all the SHs of an area provide the day-ahead load demand to the aggregator which communicates their aggregated demand to the utility. The utility then checks the availability of power at SG and provides the day-ahead power for 24 hours duration to the aggregator. The aggregator is responsible for allocating the power to each $\mathrm{SH}$ proportionate to their demand so as to maintain the load profile of SG. In addition to this power, the SH uses BESS and rooftop PV to manage its partial load demand. The power from PV panel and excess power from the utility after meeting the load requirement is stored in the BESS. Depending on the power available from the SG and the load demand at any instant, following cases arise.

Case 1: If the load demand is equal to the power supplied from SG, then load is managed using the grid's power.

Case 2: If load demand is less than the power supplied from $\mathrm{SG}$, then the BESS is utilized to fill the power deficit.

Case 3: If the instantaneous load demand in an $\mathrm{SH}$ becomes less than the power supplied from SG, then the excess power available after managing the load demand is stored in BESS.

The appliances are scheduled according to the total available power in a home using an energy management system. The schematic block diagram of the $\mathrm{SH}$ energy management system is shown in Fig. 1. As shown in the figure, the master controller (MC) is the main entity of the energy management system, which comprises a scheduler, an appliance manager, and a HAN. MC is used to control and schedule the operation(s) of the appliances intelligently. The scheduling is based on the user's preferences, power input from the SG, and the energy available in BESS. The pricing policy considered in the proposed scheme is assumed to be fixed as the case in many countries like India and UK [23], [24]. Another reason for not including dynamic pricing is because of the creation of a new re-bound peak at the grid when different energy management systems schedule the appliances simultaneously in low-price periods [8]. The various components of the $\mathrm{SH}$ energy management system are shown in Fig. 1 and their description is as follows.

Scheduler: The scheduler is the most important component of the MC, in which various algorithms are implemented to generate the appliance schedule. For each $\mathrm{SH}$, the user provides his day-ahead power consumption requirements for different time-slots to the scheduler through an user interface. The parameters such as-power allocated for SH from SG, energy available in the BESS, and user preferences are also given as inputs to the scheduler. User preferences are the ordered priority list of appliances required by the user according to his comfort. By using these parameters, an appliance schedule is generated by the scheduler. The algorithms implemented in it are discussed in Section III.

The brief working of scheduler is described as follows. 


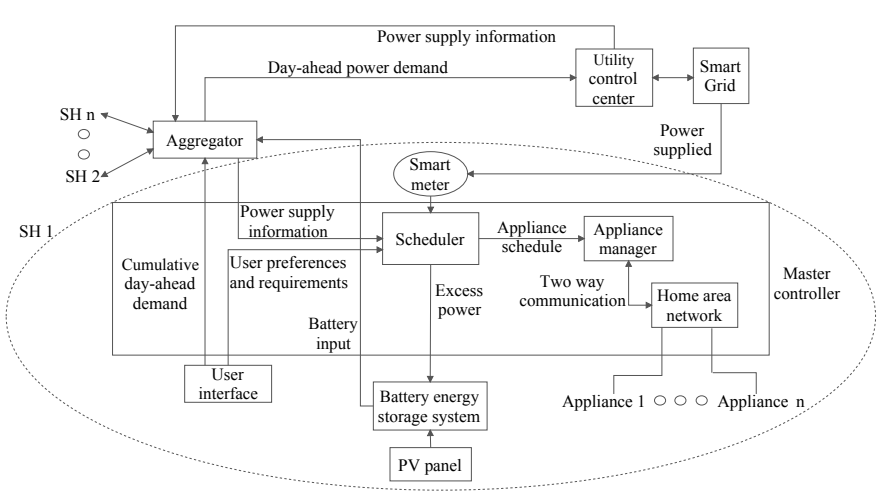

Fig. 1: Smart home energy management system.

Assume that the power available from SG and BESS at time instant $t$ are represented as $P_{g}(t)$ and $P_{b a t}(t)$ respectively, and load demand is $P_{d}(t)$, then,

Case 1: When $P_{g}(t)=P_{d}(t)$, then all the appliances in that time-slot are scheduled.

Case 2: If $P_{g}(t)<P_{d}(t)$ and $P_{g}(t)+P_{b a t}(t) \geq P_{d}(t)$, then all the appliances are scheduled by drawing the required power (i.e. $\left.P_{d}(t)-P_{g}(t)\right)$ from BESS.

Case 3: If $P_{g}(t)+P_{b a t}(t)<P_{d}(t)$, then appliances are scheduled according to Algorithms 1 and 2 as mentioned in Section III.

Case 4: If $P_{g}(t)>P_{d}(t)$, then the excess energy is stored in the BESS which acts as a load in this case.

Appliance Manager: The appliance manager controls the switching operations of the appliances using HAN corresponding to the appliance schedule generated by the scheduler.

Home area network (HAN): All the appliances inside the $\mathrm{SH}$ are connected to the MC through a HAN which may use wired or wireless technologies such as Wi-Fi, Bluetooth, and ZigBee. Using these protocols, appliances form an intra-home network to communicate with the appliance manager of SH.

Aggregator: It aggregates the load demand of all the SHs in a region and communicates it to the utility. Every $\mathrm{SH}$ sends the aggregated load demand for each time-slot to the aggregator as shown in Fig. 1. This demand is based on the load demand of different appliances used in the SH. Based on the user's preference, the load is categorized into two types: type- 1 and type- 2 load. The type- 1 load consists of non-interruptible appliances that cannot be deferred to another time-slot and whose non-functioning affects the comfort of the user. Appliances such as refrigerator, fan, TV, microwave, and $\mathrm{AC}$ can be classified into type- 1 category. On the other hand, type-2 load is interruptible and can be scheduled for later time-slots, such as washing machine and dishwasher. This categorization may vary according to the user's requirements. The average power rating of different appliances considered on the basis of their category is given in Table II.

The power required by these appliances adds up to the total power requirement for a particular SH. The day-ahead load demand of all the appliances in an SH is calculated based on the information provided by the user through the user interface. Let $P_{d}^{i}(t)$ be the total power demand at $t^{t h}$ instant (in $\mathrm{kW}$ )
TABLE II: Average power rating of different appliances

\begin{tabular}{|l|l|c|}
\hline S. No. & Appliance & Power rating (in Watt) \\
\hline \multicolumn{3}{|c|}{ Type-1 (Non-interruptible) Loads } \\
\hline 1 & Air Conditioner & 1500 \\
3 & Water heater & $1500-3000$ \\
4 & Microwave & $700-1500$ \\
5 & Electric iron & $900-1400$ \\
6 & Refrigerator & 1400 \\
7 & Electric hair dryer & 900 \\
8 & Vacuum cleaner & $200-600$ \\
9 & Television & $150-200$ \\
10 & Computer & 120 \\
11 & Fan & $50-100$ \\
12 & Tubelight & $40-60$ \\
\hline \multicolumn{2}{|c|}{ Thaver } & 15 \\
\hline 13 & Dishwasher & 1200 \\
14 & Water pump & 1000 \\
15 & Washing machine & 500 \\
\hline
\end{tabular}

of the $i^{\text {th }}$ SH which comprises both type- 1 and type-2 loads. This demand can be represented as,

$$
P_{d}^{i}(t)=\sum_{j=1}^{k} L_{j}
$$

where, $L_{j}$ is the power rating of the $j^{\text {th }}$ appliance (in $\mathrm{kW}$ ) and $k$ represents the number of appliances. For three SHs $\left\{\mathrm{SH}_{1}\right.$, $\left.\mathrm{SH}_{2}, \mathrm{SH}_{3}\right\}$, the day-ahead load demand is shown in Fig. 2. The load demand for these SHs has been taken from the homes of Patiala city of India after observing their load profiles for 24 hours.

Since solar PV panels are also installed in the SHs, their output would also be considered before estimating the power required from the SG. Suppose that $P_{p v}^{i}(t)$ is the power generated from the PV panel of $i^{t h} \mathrm{SH}$ at $t^{t h}$ time instant. Now, the total power required by this $\mathrm{SH}$ from the SG $\left(P_{r e q}^{i}(t)\right)$ is computed as follows.

$$
P_{r e q}^{i}(t)=P_{d}^{i}(t)-P_{p v}^{i}(t)
$$

For different SHs, the value of $P_{r e q}^{i}(t)$ is calculated using (2) and sent to the aggregator. For this purpose, the dayahead values of $P_{d}^{i}(t)$ is forecasted using (1) and the value of $P_{p v}^{i}(t)$ is calculated as discussed later. The aggregator then calculates the cumulative value of load demand for an entire region $\left(P_{d}^{c}(t)\right)$ and sends it to the utility.

$$
P_{d}^{c}(t)=\sum_{i=1}^{s} P_{r e q}^{i}(t)
$$

where, $s$ represents the number of SHs in a region. For example, considering a region having three SHs, Fig. 3 shows the power demand of all SHs in this region and the total power demand of the entire region, $P_{d}^{c}(t)$, which is calculated using (3).

Utility control center (UCC): It acts as an interface between the aggregator and the SG. After receiving the dayahead load information of different SHs via aggregators, UCC allocates the day-ahead power to the SHs. This information is communicated by the UCC to the respective aggregators. For example, the net power requirement of three SHs is shown in 


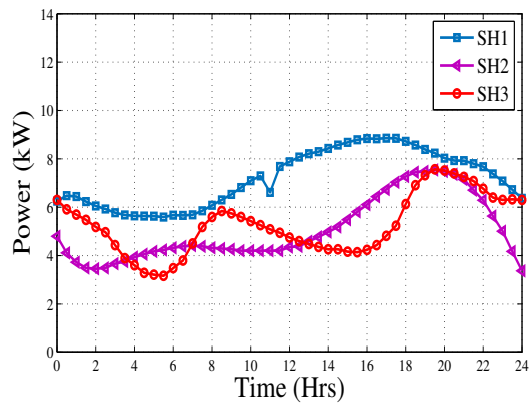

Fig. 2: Day-ahead load curve of three SHs.

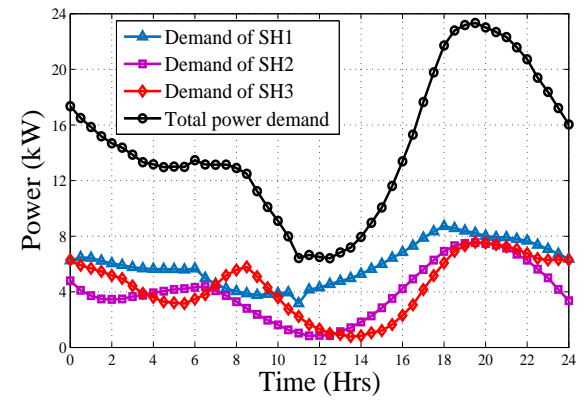

Fig. 3: Power demand of three SHs.

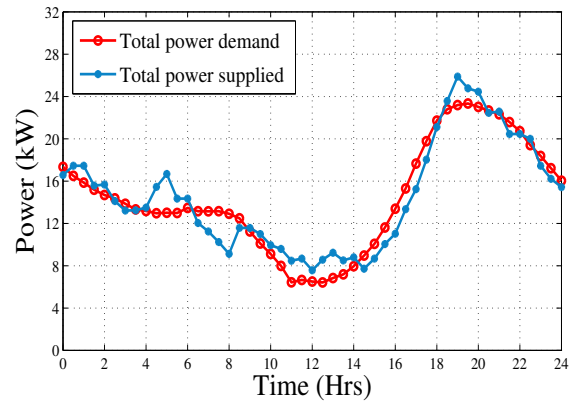

Fig. 4: Power supplied by the SG.
Fig. 3 and the power supplied by the SG to the aggregator for their total load demand is shown in Fig. 4.

The power supplied by SG is distributed amongst the SHs based on their load demands. Let $P_{g}^{t o t}(t)$ be the total power supplied by SG at $t^{t h}$ time-slot, then the power distributed to each SH is calculated using (4). For the sake of simplicity, this power is kept directly proportional to the load requirements of various SHs. However, the utility may also use different allocation policy to optimally distribute power supply to the SHs.

$$
P_{g}^{i}(t)=\left(\frac{P_{r e q}^{i}(t)}{\sum_{k=1}^{n} P_{r e q}^{k}(t)}\right) P_{g}^{t o t}(t)
$$

where, $P_{g}^{i}(t)$ is the power distributed to $i^{t h} \mathrm{SH}$ at $t$ and $P_{r e q}^{k}(t)$ depicts the power demand of $k^{t h} \mathrm{SH}$ from the total of $n \mathrm{SHs}$ (calculated using (2)).

Rooftop PV Panel: The power generated by PV panel is utilized by the respective $\mathrm{SH}$ for managing its partial load. To do so, its energy is stored in the local BESS before being utilized. The output of PV panel is modeled without performing maximum power point tracking and similar to [25]. The DC power generated by PV panel of $i^{t h} \mathrm{SH}$ at $t^{\text {th }}$ time instant $\left(P_{p v}^{i}(t)\right)$ is represented as,

$$
P_{p v}^{i}(t)=I_{f}(t) \eta_{p v} S_{a}
$$

where, $I_{f}(t)$ is the incident ray factor at $t^{t h}$ time instant. $\eta_{p v}$ and $S_{a}$ are the efficiency and surface area of the PV panel, respectively. In the steady state condition, (5) can be represented as follows.

$$
P_{r}=I_{f}^{\prime} \eta_{p v}^{\prime} S_{a}
$$

In (6), $P_{r}$ is the rated peak power, $I_{f}^{\prime}$ is the incident ray factor at maximum radiation intensity $\left(1000 \mathrm{~W} / \mathrm{m}^{2}\right)$, and $\eta_{p v}^{\prime}$ is the maximum efficiency of the PV panel. Combining (5) and (6) and considering the effect of converter, the instantaneous power produced by PV panel that can be stored in battery is represented as follows.

$$
P_{p v}^{i}(t)=P_{r} \frac{I_{f}(t) \eta_{p v}}{I_{f}^{\prime} \eta_{p v}^{\prime}} \eta_{c o n v}
$$

where, $\eta_{c o n v}$ is the efficiency of the converter and $\frac{\eta_{p v}}{\eta_{p v}^{\prime}}$ represents the thermal loss due to operating the cells above temperature of $25^{\circ} \mathrm{C}$ which is calculated as,

$$
\frac{\eta_{p v}}{\eta_{p v}^{\prime}}=1-\beta\left(T_{c}-25\right)
$$

where, $\beta$ is technical parameter defined by the manufacturer and $T_{c}(t)$ is the cell temperature at $t^{t h}$ time instant. The value of $T_{c}(t)$ is further calculated as,

$$
T_{c}(t)=T_{a}(t)+\left(\frac{T_{c}^{n o n}-20}{0.8 I_{f}^{\prime}}\right) I_{f}(t)
$$

where, $T_{a}(t)$ is the ambient temperature at $t^{t h}$ time instant and $T_{c}^{\text {non }}$ is the non-operational cell temperature whose value is provided by the manufacturer. Using (8) and (9), (7) can be written as,

$$
\begin{aligned}
& P_{p v}^{i}(t)= \\
& P_{r} \frac{I_{f}(t)}{I_{f}^{\prime}}\left[1-\beta\left[\left(T_{a}(t)+\frac{T_{c}^{n o n}-20}{0.8 I_{f}^{\prime}} I_{f}(t)\right)-25\right]\right] \eta_{c o n v}
\end{aligned}
$$

In the above equation, the value of $T_{a}$ is gathered from [26] and parameter $I_{f}$ has been forecasted using an artificial neural network as given in [27]. These values can be gathered using other resources as well. For example, the value of $T_{a}$ can be computed using any robust weather prediction module; whereas $I_{f}$ can also be forecasted using statistical models like autoregressive integrated moving average (ARIMA) [28] or by using proprietary tools like solargis [29]. Once the values of these parameters are computed, $P_{p v}^{i}$ is calculated using (10), which would be stored in the BESS. The energy in BESS after storing the power generated by PV panel is given below.

$$
\begin{gathered}
E_{\text {bat }}^{i}(t)= \\
E_{\text {bat }}^{i}(t-1)+P_{p v}^{i} \times \Delta t \\
\text { subject to } \\
E_{\text {min }}^{\text {bat }} \leq E_{\text {bat }}(t) \leq E_{\text {max }}^{\text {bat }}
\end{gathered}
$$

where, $E_{b a t}^{i}(t)$ and $E_{b a t}^{i}(t-1)$ represents the energy in BESS of $i^{t h} \mathrm{SH}$ at $t^{t h}$ and $(t-1)^{t h}$ time-slot respectively, and $\Delta t$ is the duration of time-slot. The constraint in (12) specifies that energy stored in BESS is limited by minimum $\left(E_{\text {min }}^{b a t}\right)$ and maximum ( $E_{\text {max }}^{b a t}$ ) energy storage capacity of the BESS.

Battery energy storage system (BESS): The BESS stores the energy produced by PV panel as already discussed in the previous segment. Apart from PV panel, BESS stores excess energy available in SH when power supplied by the utility is more than load demand. BESS has also been used to fill the power deficit when power supplied by the utility 
is not sufficient to cater the load demand. The effect of excess power available and power deficit in SH on BESS is discussed as follows. The energy in BESS is accumulated or de-accumulated when the power allocated by $\mathrm{SG}$ is not equal to the load demand of $\mathrm{SH}\left(P_{g}^{i}(t) \neq P_{d}^{i}(t)\right)$. In this scenario, the BESS either charges $\left(P_{g}^{i}(t)>P_{d}^{i}(t)\right)$ or discharges $\left(P_{g}^{i}(t)<P_{d}^{i}(t)\right)$ at any particular time-slot.

The energy in battery after charging is given by,

$$
E_{b a t}^{i}(t)=\left(E_{b a t}^{i}(t-1)+\left(P_{g}^{i}(t)-P_{d}^{i}(t)\right) \Delta t\right) \eta_{\text {rect }}
$$

where, $\eta_{\text {rect }}$ is the efficiency of the rectifier.

The energy in battery after discharging is given by,

$$
E_{b a t}^{i}(t)=\frac{E_{b a t}^{i}(t-1)+\left(P_{g}^{i}(t)-P_{d}^{i}(t)\right) \Delta t}{\eta_{i n v}}
$$

where, $\eta_{i n v}$ is the efficiency of the inverter. These equations are also subjected to the constraint specified in (12). The BESS is not charged if it has reached its maximum capacity. Moreover, it stops supplying the energy on reaching its minimum capacity. It is to be noted that the losses due to leakage current and charging/discharging efficiency of the battery are assumed to be negligible and have not been considered in this paper.

\section{Design of Scheduler Using Heuristic ApProAch}

This section formulates the appliance scheduling problem on the basis of a heuristic approach and also highlights the working methodology of the proposed scheduling scheme. Different algorithms have been proposed in this scheme which are distributed in nature, i.e., they work for individual homes at the scheduler component of the energy management system.

\section{A. Problem formulation}

The objective of the appliance scheduling is to schedule the devices of SHs in each time-slot so as to balance their load profiles. The scheduling of devices is done according to the grid constraints, the scheduled end-times of the appliances, and user's preference order. This optimization problem can be represented as,

$$
\begin{aligned}
& \text { Minimize } \\
& \qquad P_{e x}(t)=P_{g}(t)+P_{p v}(t)-P_{d}(t) ; \forall t
\end{aligned}
$$

where, $P_{e x}(t)$ is the extra power available in an $\mathrm{SH}$ at any instant $t$; and $P_{g}(t), P_{p v}(t)$, and $P_{d}(t)$ represents the power supplied by the grid, power available from the PV panel, and power demand for one $\mathrm{SH}$ at $t$, respectively. To achieve the given objective, the value of these variables is required for every time-slot. The values of $P_{g}(t)$ and $P_{d}(t)$ are calculated using (4) and (1) respectively; while the value of $P_{p v}(t)$ is computed using (10). The objective of (15) is to schedule the devices so as to minimize the value of $P_{e x}(t)$ for a day. For this purpose, the complete day has been divided into 96 time-slots of 15 minutes duration each (i.e. $\Delta t=15 \mathrm{~min}$ ). For all the time-slots, (15) gives the near-optimal solution by scheduling the maximum number of appliances subject to the availability of power. The excess power is minimized either by rescheduling the appliances in that time-slot or by storing it in the BESS. There are few theoretical bounds that exist on the optimization problem which are given below.

$$
\begin{gathered}
P_{e x}(t) \geq 0 ; \forall t \\
D L^{t_{i}} \text { scheduled according to } U P^{t_{i}} ; \forall i \\
0 \leq P_{p v}(t) \leq P_{r} ; \forall t \\
\sum_{t=1}^{h}\left(t+n_{r t}^{d}-1\right) . \Delta t \leq E T_{d} ; \forall d
\end{gathered}
$$

The constraint given in (16) makes certain that rescheduling of appliances is done when the load demand in an SH is greater than the power supplied by SG and power available in BESS. Constraint (17) ensures that the user priority is taken into consideration while rescheduling the appliances. In (17), $D L^{t_{i}}$ contains the list of appliances specified by the user in the $i^{\text {th }}$ time-slot; and $U P^{t_{i}}$ contains the user-specified priority of these appliances. This priority is set by the user beforehand through the user interface as shown in Fig. 1. Constraint (18) specifies that the power generated by PV panel is limited by the rated PV panel capacity. The constraint (19) means that the operation of any device $d$ should be finished before its specified end-time in list $E T_{d}$. The value of $h$ specifies the maximum value of time-slots and $n_{r t}^{d}$ specifies the number of time-slots for which device $d$ needs to be run in order to finish its operation.

The proposed scheme is based on a heuristic approach and it splits the appliance scheduling problem for a day into subproblems for different time-slots to find the initial schedule. As the heuristic approach is best suited for finding near-optimal solutions when subjected to constraints on theoretical bounds [30], [31]. Thus, the proposed scheme also gives an nearoptimal appliance schedule for every time-slot when bounded by the constraints specified in (16)-(19).

\section{B. Methodology}

The flowchart of the proposed scheduling scheme for one $\mathrm{SH}$ is shown in Fig. 5 and its working is described as follows. Initially, the power generated by PV panel is stored in the BESS as shown in the figure. The information regarding the load demand and power available in BESS at time instant $t$ $\left(P_{b a t}(t)\right)$ is sent to the aggregator, which communicates the power required by the SH from SG (i.e. $P_{d}(t)-P_{b a t}(t)$ ) to the UCC. The UCC supplies $P_{g}(t)$ amount of power based on the demand of SH. If for a given time-slot $P_{g}(t) \geq P_{d}(t)$, then all the appliances are scheduled and the extra energy (if any) is stored in the BESS. If $P_{g}(t)+P_{b a t}(t) \geq P_{d}(t)$, then all the devices in that time-slot are scheduled. Otherwise, algorithm 1 is used for the scheduling of appliances. This algorithm also takes user's preferences into account while scheduling. Once the appliance schedule is generated, if any unscheduled appliance is switched on, then algorithm 2 is used to handle this instantaneous load change. The purpose of these algorithms is to achieve the objective function given in (15) while complying with the constraints given in (16)-(19). 


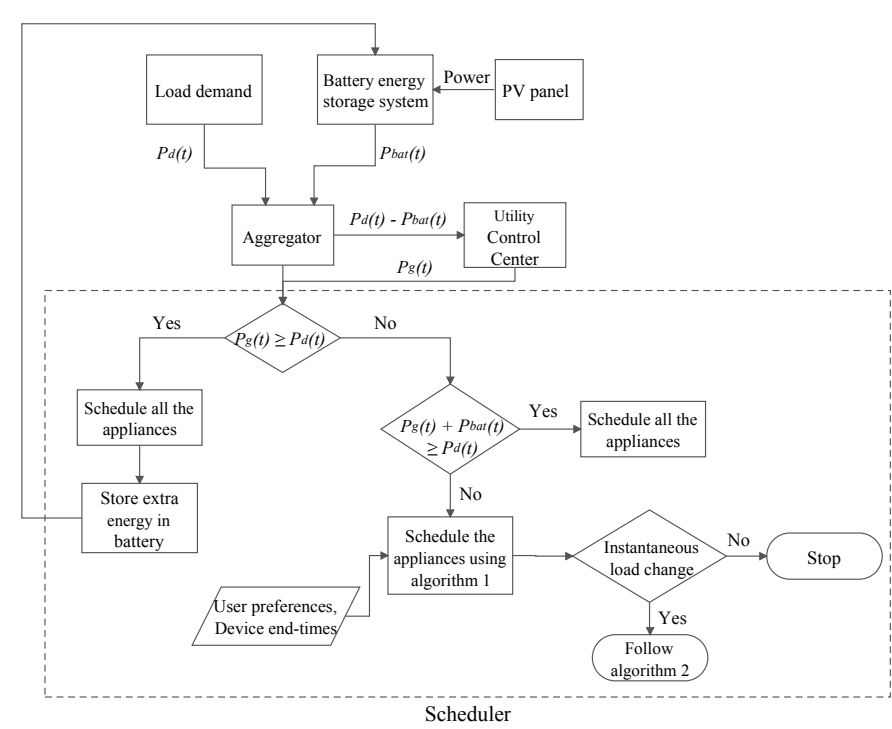

Fig. 5: Flowchart of the proposed scheme.

1) Scheduling of appliances: After the initial schedule is specified by the user, the appliances are scheduled according to algorithm 1 based on the user's priority and specified end time of appliances.

The working of this algorithm is as follows. Initially, the load requirement of the user is calculated using (1). For every time-slot, user-defined schedule is divided into two lists $\left(L_{1}^{t_{i}}\right.$, $L_{2}^{t_{i}}$ ), which store the information regarding type-1 and type-2 appliances, respectively. A structure $\left(D_{i}^{t}\right)$ is created to store these lists and the value of $P_{e x}(t)$ is computed from (15). (If a device has to run over more than one time-slot, then it's information is copied into the lists for subsequent time-slots.) $D_{i}^{t}$ is then sorted in descending order according to value of $P_{e x}(t)$. A function, $\operatorname{SORT}(\mathrm{L}, \mathrm{P})$, is used for sorting the lists $L_{1}^{t_{i}}$ and $L_{2}^{t_{i}}$ according to user preferences (stored in list $U P^{t_{i}}$ ). Then, the list $L$ is re-arranged according to the end times of the appliances given in list $E T_{d}$. For example, if an appliance has the end time of $8 \mathrm{pm}$, then it is scheduled before the appliance which has an end time of $10 \mathrm{pm}$. It is to be noted that the end time of appliances which can be scheduled at any time of the day, is set to the highest value. Now, if the available power in an SH is greater than the load requirement, then all the appliances are scheduled and extra energy (if any) is stored in the BESS (if $P_{b a t}(t) \leq P_{\max }^{b a t}$ ). Otherwise, the last item from the second list (i.e. $L_{2}^{t_{i}}$ ) is removed and it is scheduled at the first instance of list $L_{2}$ of structure $D_{i}^{t}$. The removed appliances are placed in a queue $(Q)$ when there is no time-slot for which $P_{e x}(t)>0$. This process is repeated until load becomes less than the available power in that timeslot, or list $L_{2}^{t_{i}}$ becomes empty. If list $L_{2}^{t_{i}}$ is empty, then the same procedure is repeated for list $L_{1}^{t_{i}}$ until load complies with the available power. It may be the case that $P_{e x}(t)$ becomes available in different time-slots after rescheduling of the appliances. In such a scenario, the appliances placed in $Q$ are scheduled in these time-slots according to the best-fit approach. The appliances (in $Q$ ) that cannot be accommodated are not scheduled for that day.

Time complexity: Time complexity of this algorithm is

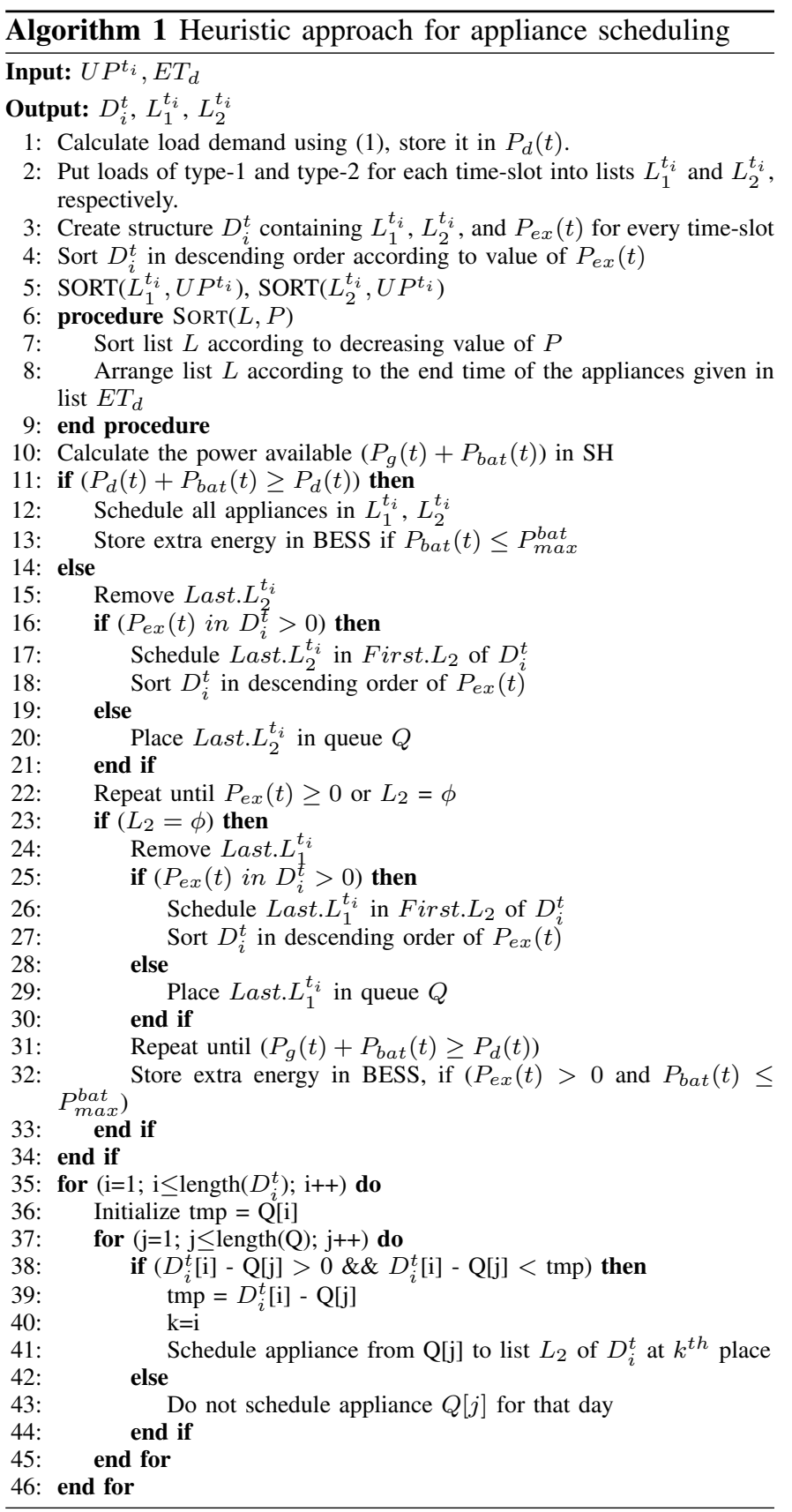

calculated as follows. Step 2 and 3 takes $O(n)$ time. The sorting procedure is performed using the merge sort which takes $O(n \log n)$ time. Step 18 is again a sorting step, which takes $O(t \log t)$ time where, $t$ is the number of time-slots. For worst case scenario, step 22 would take $O(n . t)$ time. Step 27 takes $O(t \log t)$ time and step 31 takes $O(n . t)$ time. Steps 38 to 41 takes $O(t . q)$ for worst case scenario, where $q$ is the number of appliances in queue $Q$. All other steps takes unit time. Considering all of these, the overall time complexity (TC) is computed as follows.

$$
\begin{aligned}
& T C=O(n)+O(n \log n)+O(t \log t)+O(n . t)+O(t . q) \\
& \Rightarrow T C=O(n \log n)
\end{aligned}
$$

Space complexity: Initially, there are four lists and a structure namely $U P^{t_{i}}, E T_{d}, L_{1}^{t_{i}}, L_{2}^{t_{i}}$, and $D_{i}^{t}$ which would 
take space of $n, n, n, n$, and $3 n$ respectively. Lists $L$ and $P$ are also used in the algorithm in SORT function, which takes space of $n$ each. Queue $Q$ would take $q$ space. So, the overall space complexity (SC) is computed as follows.

$$
\begin{aligned}
& S C=O(n)+O(q) \\
& \Rightarrow S C=O(n)
\end{aligned}
$$

Example 1: Suppose that there are three time-slots, $\left\{T_{1}, T_{2}, T_{3}\right\}$ for which the scheduling is to be done. The lists of appliances $\left(L_{1}, L_{2}\right)$ sorted according to the user's preference is shown in Table III. (All the values in this table are in $\mathrm{kW}$. The values of $P_{g}(t)$ and $P_{b a t}(t)$ are taken as constant having $8 \mathrm{~kW}$ and $2 \mathrm{~kW}$ of power, respectively in each time-slot and the end times of the appliances are considered flexible for the sake of simplicity.)

TABLE III: List of appliances in different time-slots (Example 1)

\begin{tabular}{|l|l|l|l|l|l|l|}
\hline \multirow{2}{*}{ Time-slot } & \multicolumn{3}{|c|}{ Initial } & \multicolumn{3}{c|}{ Final } \\
\cline { 2 - 7 } & $L_{1}^{t_{1}}$ & $L_{2}^{\iota_{1}}$ & $P_{d}(t)$ & $L_{1}^{t_{1}}$ & $L_{2}^{t_{1}}$ & $P_{d}(t)$ \\
\hline \multirow{3}{*}{$T_{1}$} & $D_{11}=2$ & $D_{14}=4$ & & $D_{11}=2$ & $D_{14}=4$ & \\
& $D_{12}=3$ & $D_{15}=3$ & 13 & $D_{12}=3$ & & 10 \\
& $D_{13}=1$ & & & $D_{13}=1$ & & \\
\hline \multirow{3}{*}{$T_{2}$} & $D_{21}=2$ & $D_{24}=2$ & & $D_{21}=2$ & $D_{24}=2$ & \\
& $D_{22}=3$ & $D_{25}=1$ & 9 & $D_{22}=3$ & $D_{25}=1$ & 9 \\
& $D_{23}=1$ & & & $D_{23}=1$ & & \\
\hline \multirow{3}{*}{$T_{3}$} & $D_{31}=2.5$ & $D_{34}=2$ & & $D_{31}=2.5$ & $D_{15}=3$ & \multirow{2}{*}{$\begin{array}{l} \\
\end{array}$} \\
& $D_{32}=1.5$ & $D_{35}=0.5$ & 7 & $D_{32}=1.5$ & $D_{34}=2$ & 10 \\
\hline
\end{tabular}

The value of $P_{e x}(t)$ is then calculated, which comes out to be $\{-3,1,3\}$ for $\left\{T_{1}, T_{2}, T_{3}\right\}$, respectively. Now, for $T_{1}$, according to the algorithm, appliance $D_{15}$ is removed from list $L_{2}$ and scheduled at first instance in list $L_{2}$ of $T_{3}$ as it has maximum $P_{e x}(t)$. This rescheduling balances the demand and supply in time-slots $T_{1}$ and $T_{3}$. For $T_{2}$, the extra energy is stored in the BESS for later use. The final schedule of appliances as given by the algorithm is also shown in Table III.

2) Instantaneous load balancing: Algorithm 1 gives the appliance schedule that the user needs to follow. But, if the user switches on any unscheduled appliances in a particular time-slot, then algorithm 2 is used to update the schedule for remaining time-slots (as the algorithm works in real-time, thus it cannot schedule a load in time-slot that has already elapsed). It is assumed that if the user switches on any unscheduled appliance, then it is required urgently and its priority is set to the maximum value.

The working of the algorithm 2 is as follows. If any new appliance (say $D_{\text {new }}$ ) is switched on, then its priority is set to the maximum and it is placed at the first instance of list $L_{1}^{t_{i}}$. Now, if the load demand at that instant is less than the available power, then all the appliances are run in parallel with this new appliance. The extra energy, if available, is stored in the BESS. Otherwise, the last appliance from list $L_{2}^{t_{i}}$ is removed and it is scheduled at first instance of list $L_{2}$ in structure $D_{i}^{t}$. $D_{i}^{t}$ is then sorted in descending order according to the value of $P_{e x}(t)$ for the remaining time-slots. If the value of $P_{e x}(t)<0$ for all $t$, the appliance removed from list $L_{2}^{t_{i}}$ is not scheduled during that day. If the list $L_{2}^{t_{i}}$ is empty, then the appliances from list $L_{1}^{t_{i}}$ are removed and scheduled in a similar fashion. This process is repeated until the load requirement for that time-slot

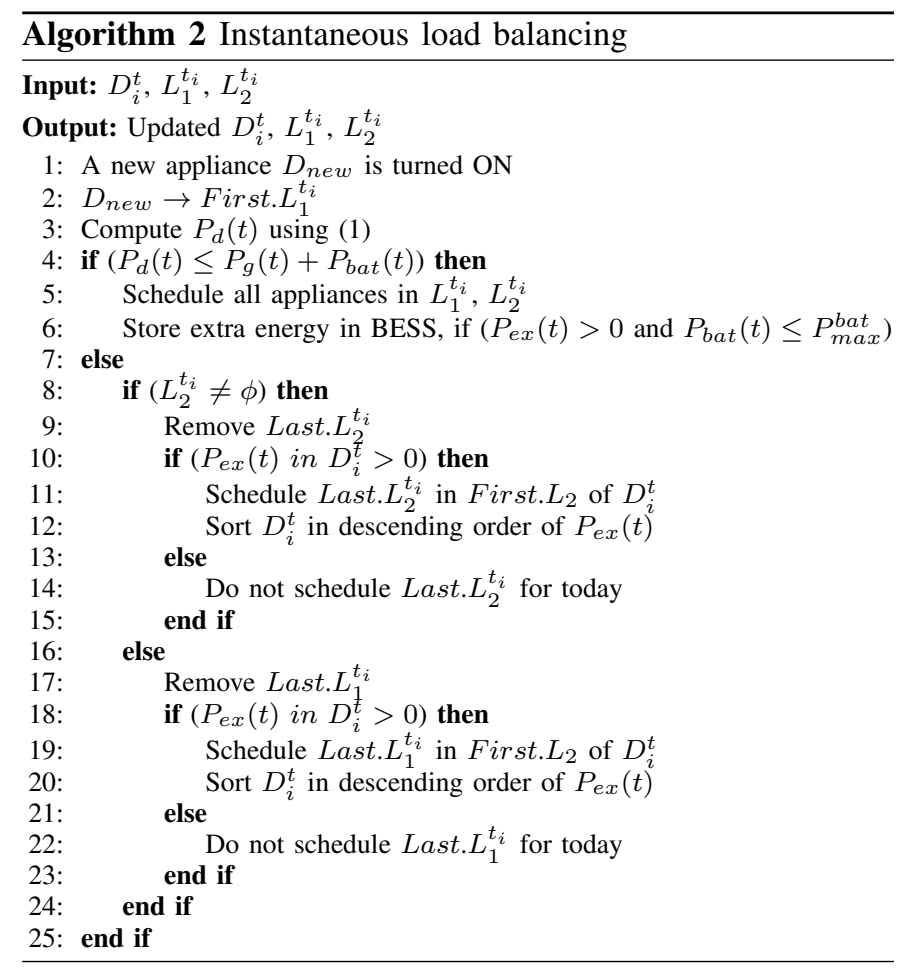

is less than the available power. In this way, the instantaneous load requirements of an $\mathrm{SH}$ are managed in real-time using the proposed scheme.

Time complexity: The time complexity of this algorithm is computed as follows. Steps 4 to 6 takes $O(n . t)$ time and step 12 takes $O(t \log t)$. Step 20 takes $O(t \log t)$ time. All other steps take unit time to compute. Hence, the overall time complexity of this algorithm is given as follows.

$$
\begin{aligned}
& T C=O(n . t)+O(\text { tlogt }) \\
& \Rightarrow T C=O(n . t)
\end{aligned}
$$

Space complexity: There are two lists and one structure, which are used in this algorithm. Lists $L_{1}^{t_{i}}, L_{2}^{t_{i}}$ takes $n$ space each and structure $D_{i}^{t}$ takes $3 n$ space. So, total space complexity of this algorithm is as follows.

$$
\Rightarrow S C=O(n)
$$

Example 2: In this example, the power available in a particular time-slot $(T)$ is taken as $10 \mathrm{~kW}$ and the initial schedule generated by the algorithm 1 is as shown in Table IV (All the values are taken in $\mathrm{kW}$ ).

TABLE IV: List of appliances in different time-slots (Example 2)

\begin{tabular}{|l|l|l|l|l|l|l|}
\hline \multirow{2}{*}{ Time-slot } & \multicolumn{3}{|c|}{ Initial } & \multicolumn{3}{c|}{ Final } \\
\cline { 2 - 7 } & $L_{1}^{t}$ & $L_{2}^{t}$ & $P_{d}(t)$ & $L_{1}^{t}$ & $L_{2}^{t}$ & $P_{d}(t)$ \\
\hline \multirow{T}{*}{$T$} & $D_{1}=2$ & $D_{5}=1.5$ & & $D_{n e w}=2$ & & \\
& $D_{2}=1$ & $D_{6}=0.5$ & 10 & $D_{1}=2$ & & 10 \\
& $D_{3}=3$ & & & $D_{2}=1$ & & \\
& $D_{4}=2$ & & & $D_{3}=3$ & & \\
& & & & $D_{4}=2$ & & \\
\hline
\end{tabular}

Now, let us assume that an appliance, $D_{\text {new }}$ of rating 2 $\mathrm{kW}$, is switched on by the user instantly in this time-slot. Then, according to the algorithm, the appliance $D_{6}$ would be removed from list $L_{2}$. Since the load demand is still more 
than $10 \mathrm{~kW}$; therefore, appliance $D_{5}$ is also removed from list $L_{2}$ and thereby making the load demand equal to the available power in $T$. The final load demand at time-slot $T$ is as shown in Table IV. Appliances $D_{6}$ and $D_{5}$ are scheduled in the succeeding time-slots with less load requirements.

\section{Simulation RESUlts AND Discussion}

To evaluate the proposed scheme, the simulation scenario considers two case studies. The first case study presents the detailed results for three households and the second one extends the proposed scheme for a large residential community.

\section{A. Case study 1 - Individual homes}

The 24 hour load profile of three homes which are equipped with a BESS, has been considered for this case study. The devices in the homes send and receive signals to and from the controller located in the home as shown in Fig. 6. The controller sends the control signals to the devices using antenna operating at $855 \mathrm{MHz}$ frequency with the help of a RF transceiver (connected to the controller) as shown in Fig. 6. The devices are equipped with the RF modules which receives the control signals (ON/OFF) from the controller and switch it ON/OFF using the electromechanical relay. The simulation is carried out in MATLAB R2014a and the parameters used in the simulation are described in Table V.

The complete day has been divided into time-slots of 15 minutes each. The maximum capacity of the PV panel is 4 $\mathrm{kWh}$. The rated capacity of the BESS is taken as $8 \mathrm{kWh}$, while $E_{m i n}^{b a t t}$ and $E_{m a x}^{b a t t}$ are assumed to be $10 \%$ and $100 \%$, respectively. Although, the actual operational limits of battery may vary depending on the type of battery and manufacturer. The degradation of battery's capacity and lifespan due to constant use of battery have been neglected for the sake of simplicity. The battery size is taken by keeping in view of the load profile of the homes and cost of the battery. It has been judiciously chosen so that it can store the excess power supply by the grid and also temporarily meet the energy demand of homes.

TABLE V: Simulation parameters

\begin{tabular}{|l|l|}
\hline Parameters & Values \\
\hline Battery Capacity & $8 \mathrm{kWh}$ \\
$P_{r}$ & $4 \mathrm{~kW}$ \\
Initial energy in BESS & $3 \mathrm{kWh}$ \\
$\triangle t$ & $15 \mathrm{~min}$ \\
$E_{\text {min }}^{\text {bint }}$ & $0.8 \mathrm{kWh}$ \\
$E_{\text {mat }}^{\text {max }}$ & $8 \mathrm{kWh}$ \\
$\eta_{\text {conv }}$ & 0.9 \\
$\eta_{\text {inv }}$ & 0.95 \\
$\eta_{\text {rect }}$ & 1 \\
\hline
\end{tabular}

Initially, the power is distributed amongst $\mathrm{SHs}$ based on their load demands as shown in Fig. 7. In this figure, at 08:00 hrs, the power distributed to $\left\{S_{1}, S H_{2}\right.$, and $\left.S H_{3}\right\}$ is $\{3.53$ $\mathrm{kW}, 2.35 \mathrm{~kW}$, and $3.24 \mathrm{~kW}$, respectively which is calculated using (4). In this equation, the power supplied by the SG is $9.1 \mathrm{~kW}$ (as shown in Fig. 4) and the values of $\alpha_{1}, \alpha_{2}$, and $\alpha_{3}$ are $\{1.01,0.67$, and 0.93$\}$ respectively. These values are proportionate to the initial load demand of the SHs which is $\{6.08 \mathrm{~kW}, 4.04 \mathrm{~kW}$, and $5.57 \mathrm{~kW}\}$, respectively as shown in Fig. 2.

As the main focus of this paper is to schedule the appliances for individual SHs, hence the subsequent results are presented for a single SH. For instance, the power demand of $S H_{1}$ from SG and the power supplied by SG in lieu of this demand is shown in Fig. 8. As inferred from this figure, the power required from SG is less than the initial load demand during 06:00 hrs to 18:00 hrs. It is due to the fact that the PV panel has been utilized in SH to cater the partial load demand, which eases the burden on SG during these hours. For example, at 12:00 hrs, the initial load demand is $7.88 \mathrm{~kW}$ and the power generated by the PV panel at this instant is $3.57 \mathrm{~kW}$. Therefore, the power required from the $\mathrm{SG}$ at this instant comes down to $4.31 \mathrm{~kW}$. However, the power supplied by the SG, at certain times, is more than that of the power demand. For example, at 12:00 hrs, the SG provides $5.01 \mathrm{~kW}$ of power but the required power is $4.31 \mathrm{~kW}$. The consequent surplus energy is stored in the BESS, which can be utilized when needed. The BESS also stores the instantaneous power generated by PV panel (as shown in Fig. 9) in accordance with (11). In addition to it, Fig. 9 also shows the instantaneous power available in the BESS without considering the PV panel. This available power depends on the initial schedule of appliances generated by the scheduler using algorithm 1 (as shown in Fig. 12) and power supplied from the SG (as shown in Fig. 8).

The total energy in the BESS after considering the available power and power generated by PV panels is as shown in Fig. 10. The initial energy available in the BESS at the start of the day is assumed to be $3 \mathrm{kWh}$ which remained unused from the previous day. As the BESS is utilized for scheduling of the appliances, the energy present in it changes accordingly. Fig. 11 shows the change in energy of the BESS due to charging or discharging with respect to the preceding timeslot (in accordance with (13) and (14)). For example, 0.235 $\mathrm{kWh}$ (i.e., $\frac{0.94}{4} \mathrm{kWh}$ ) of energy is stored at 04:45 hrs (as load demand is $6 \mathrm{~kW}$ and power available from SG is $6.94 \mathrm{~kW}$ ). In Fig. 11, the negative power of y-axis means that the power is taken out from the BESS while the positive power means that the power is supplied to the BESS.

The appliance schedule is managed according to the energy in the BESS as well as the power supplied by the SG as discussed in Section III-B1. The appliance priority set beforehand by the user of $S H_{1}$ is also utilized in algorithm 1 to generate the appliance schedule. Fig. 12 shows the initial power demand of the user and the updated power demand to manage the appliance schedule as generated by algorithm 1 . Two inferences can be drawn from this figure. First, all the appliances have been scheduled when the power supplied by SG and the energy available from the BESS is sufficient to meet the load demand. Second, when the available power is less than the power demand, the appliances are rescheduled across other time-slots having excess power. It is done so that the power consumption is in accordance with the power available. These appliances are rescheduled based on the user specified priority. For example, the low priority appliances in $15: 45 \mathrm{hrs}$ are shifted to $11: 30 \mathrm{hrs}$, where the available 


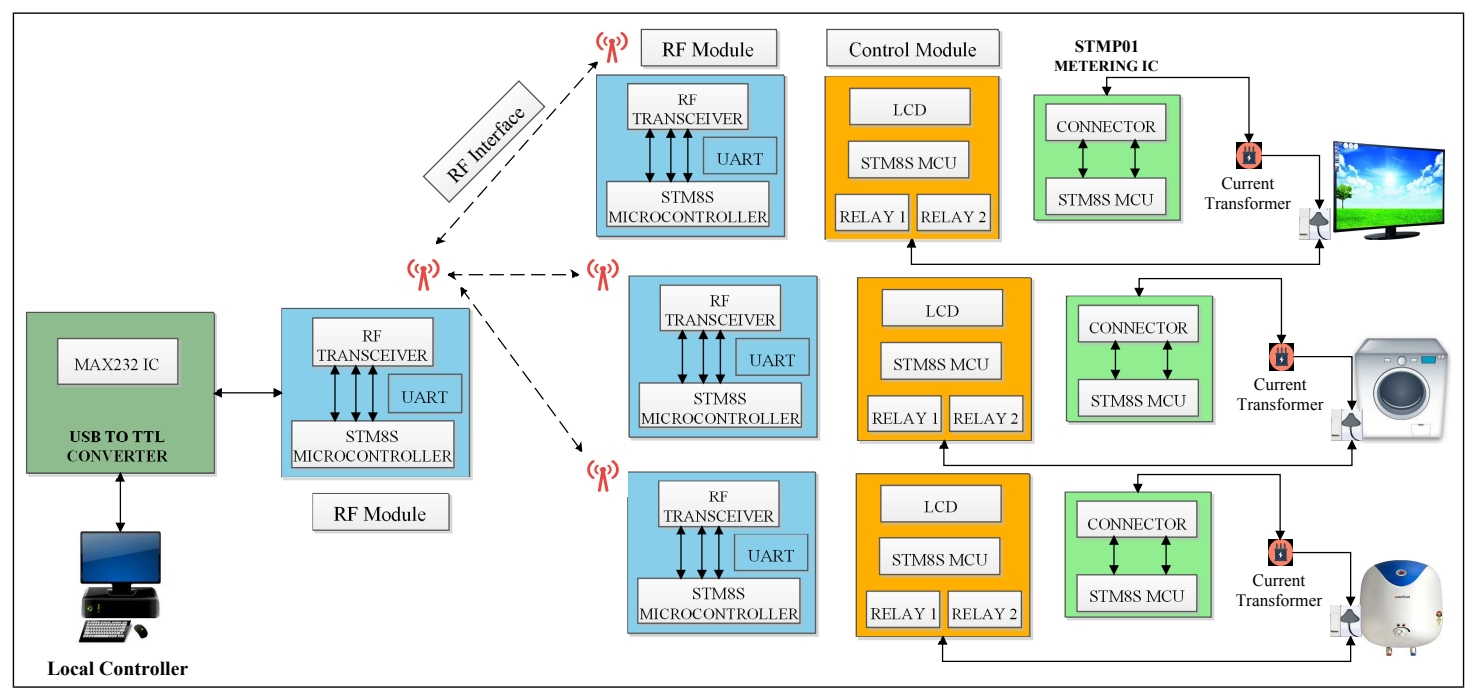

Fig. 6: Simulation setup of a home.

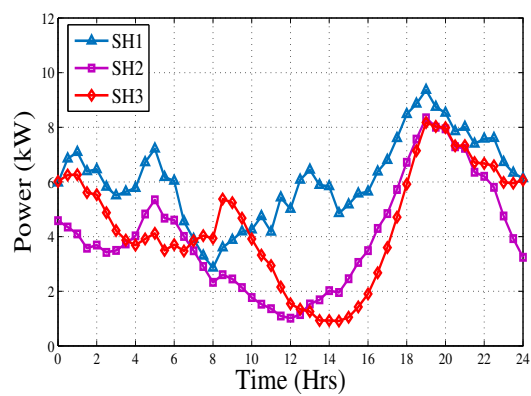

Fig. 7: Power distributed to different SHs.

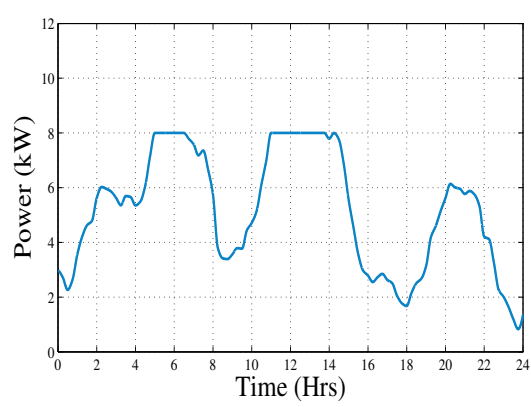

Fig. 10: Total energy in the BESS.

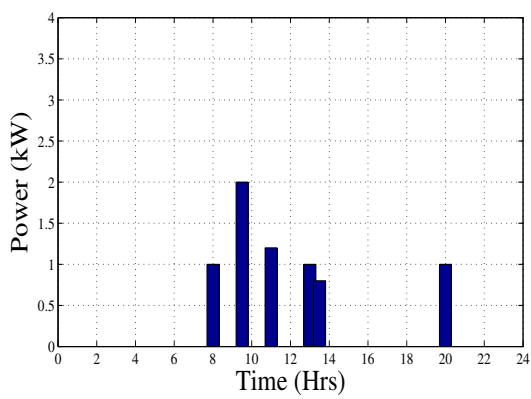

Fig. 13: Instantaneous load demand in different time-slots.

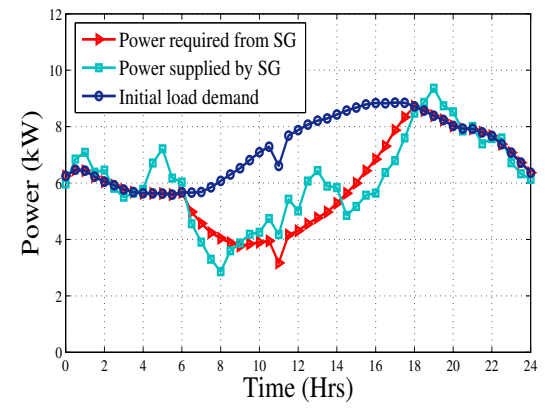

Fig. 8: Power supplied by SG to $S H_{1}$.

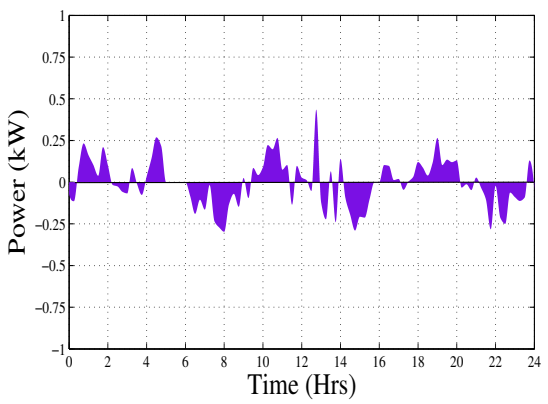

Fig. 11: Change in energy of the BESS.

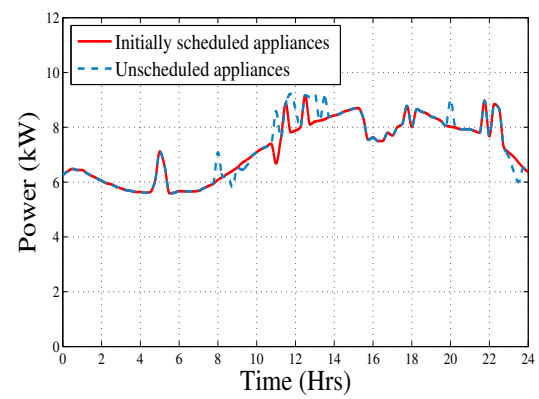

Fig. 14: Power required to manage unscheduled appliances.

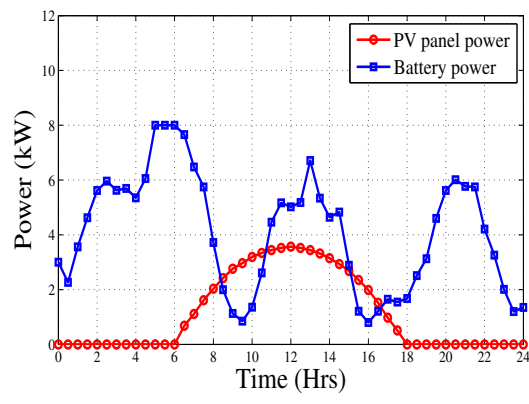

Fig. 9: Instantaneous power available in the BESS.

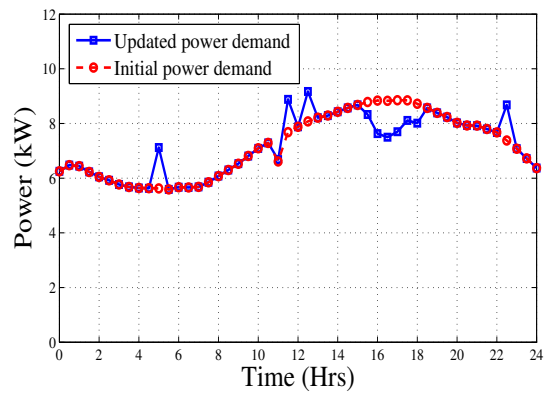

Fig. 12: Power required to manage load demand.

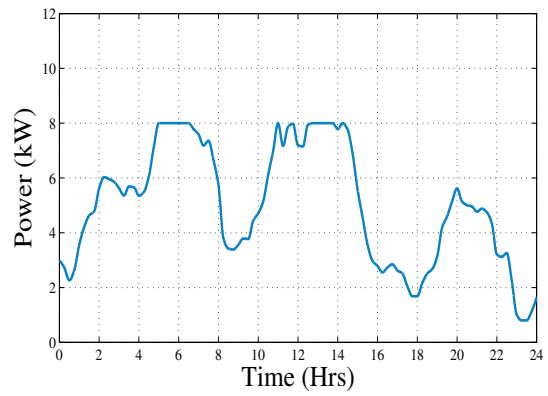

Fig. 15: Energy available in BESS after considering instantaneous load. 

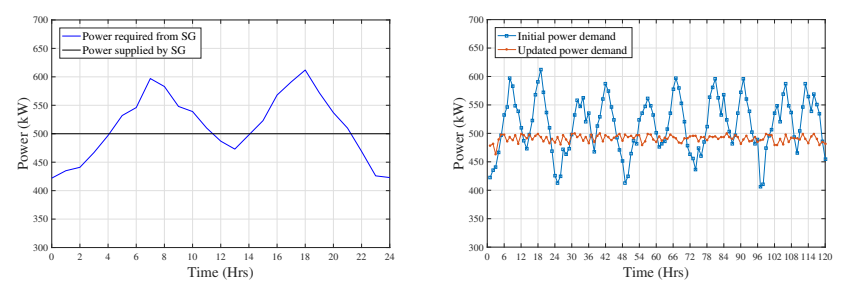

Fig. 16: Load demand of theFig. 17: Power required to manage residential community. residential community.

power is maximum. In fact, the proposed scheme also helps in reducing the power dissipation to a minimum value. It does so by scheduling the appliances to time-slots when power from the SG and power generated by PV panel exceeds the BESS capacity after meeting the load.

There can also occur a condition where the load changes instantaneously when the user switches on the unscheduled appliances due to urgency as shown in Fig. 13. In such a scenario, the instantaneous load demand is managed using algorithm 2. As seen in Fig. 13, the user requires $1 \mathrm{~kW}$ of load (TV, Fan, and Microwave) at 08:00 hrs, $2 \mathrm{~kW}$ at 09:30 hrs, $1.2 \mathrm{~kW}$ at $11: 00 \mathrm{hrs}, 1 \mathrm{~kW}$ at 13:00 hrs, $0.8 \mathrm{~kW}$ at 13:30 hrs, and $1 \mathrm{~kW}$ at 20:00 hrs. This load is managed by the available power in these time-slots. If this power is able to fulfill the new load demand, then all the appliances are scheduled in same time-slots. Otherwise, low priority appliances are rescheduled to accommodate the new load as discussed in Section III-B2. For example, the available power at 08:00 hrs is sufficient to accommodate the new appliances, thus these are scheduled concurrently with the old ones. But, the power at 09:30 hrs is not sufficient, so the appliances of low priority (having power $1.4 \mathrm{~kW}$ and 0.8 $\mathrm{kW}$, respectively) are rescheduled to subsequent time-slots of 11:45 hrs and 12:00 hrs, respectively. A similar process is repeated for all other time-slots. The updated power demand after accommodating these unscheduled appliances is shown in Fig. 14. After scheduling of the instantaneous load, the energy stored in the BESS has also changed accordingly, which is shown in Fig. 15.

\section{B. Case study 2 - Large residential community}

This case study considers 50 homes in a residential community to perform the simulation study. The data of these homes is taken from [32] for the month of June and distributed to various appliances according to the usage pattern specified in [33]. Each home is assumed to have the BESS with an initial battery capacity of $2 \mathrm{kWh}$ and the weather is assumed to be sunny during the considered period for the sake of simplicity. It is to be noted that these values and conditions would vary according to the individual homes in the real world. The power supply from the grid is considered to be a constant of 500 $\mathrm{kW}$ which is distributed to every household using (4). By extending the proposed scheduler presented in Section III on the basis of available power available from grid and BESS, the consolidated results for the whole residential community for load scheduling are depicted in Figs. 16 and 17.
Fig. 16 shows the initial and updated power demand of the whole community for a complete day before and after using the designed scheduler in the homes. It is to be noted that the decline in the initial load demand during 10:00 to 14:00 hrs is because it also takes into account the local power generated by the PV panel during this period. It can be inferred from this figure that there is a mismatch between the power supply and initial load demand during the complete day. Thus, algorithm 1 is used for every home to generate the initial appliance schedule on the basis of available power so that this mismatch can be minimized. As a result, the load demand in the individual homes is updated which updates the overall load demand of the whole community. This is illustrated in Fig. 17 which depicts the overall power supplied before and after using the proposed scheduling scheme for five calendar days of the month. The overall gains over the illustrated period are visible in terms of the load rescheduling according to the available power in a home. The results of this case study also prove that the proposed scheme is scalable to apply on any number of SHs on any given day.

\section{CONClusion}

With the advancements in SHs, the load fluctuations in grids can be reduced so that the stability of the grid is maintained. In order to do so, the load demand of SHs needs to comply with the power supplied by the utility. In this paper, we have considered the use of PV panels and BESS in addition to the power supplied by the grid, to manage the load demands of SHs. Unlike other approaches, the proposed scheme handles the power requirement of SHs by scheduling the appliances while complying with the power supplied by the utility. For this purpose, the power supplied from the SG is distributed amongst the SHs based on their power requirements. The appliances are then scheduled according to the available power and user's priority. The interruptible load is shifted to the time instances having less power requirement. In addition to it, instantaneous load change is also catered by rescheduling the appliances of less preference in other time-slots. Moreover, the excess energy available after the scheduling of appliances is stored in the BESS. Simulation results show the effectiveness of the proposed scheme to handle the load requirements of SHs with respect to the power supplied by the utility.

In the future, a centralized controller would be designed so as to decrease the required infrastructure cost of the proposed scheme while considering the scalability of the algorithms. Moreover, the results would be evaluated with different weather conditions, different building characteristics, and explicit modeling of appliances to test the effectiveness from the grid perspective.

\section{ACKNOWLEDGMENT}

The research work carried out in this paper has been partly sponsored by Council of Scientific and Industrial Research, India (Sanction No. 09/677(0025)/2015-EMR-I and 22(0717)/16/EMR-II). 


\section{REFERENCES}

[1] M.L .Tuballa and M.L. Abundo, "A review of the development of Smart Grid technologies," Renewable and Sustainable Energy Reviews, vol. 59, pp. 710-725, 2016.

[2] A. Safdarian, M. F-Firuzabad, and M. Lehtonen, "Benefits of Demand Response on Operation of Distribution Networks: A Case Study," IEEE Systems Journal, vol. 10, no. 1, pp. 189-197, 2016.

[3] A. Zipperer, P. A. A. Young, S. Suryanarayanan, R. Roche, L. Earle, D. Christensen, P. Bauleo, and D. Zimmerle, "Electric Energy Management in the Smart Home: Perspectives on Enabling Technologies and Consumer Behavior," Proceedings of the IEEE, vol. 101, no. 11, pp. 2397-2408, 2013.

[4] M. Li and H. J. Lin, "Design and Implementation of Smart Home Control Systems Based on Wireless Sensor Networks and Power Line Communications," IEEE Transactions on Industrial Electronics, vol. 62, no. 7, pp. 4430-4442, 2015.

[5] D. M. Han and J. H. Lim, "Smart Home Energy Management System using IEEE 802.15.4 and ZigBee," IEEE Transactions on Consumer Electronics, vol. 56, no. 3, pp. 1403-1410, 2010.

[6] D. Li and S. K. Jayaweera, "Distributed Smart-Home Decision-Making in a Hierarchical Interactive Smart Grid Architecture," IEEE Transactions On Parallel And Distributed Systems, vol. 26, no. 1, pp. 75-84, 2015.

[7] I. Zenginis, J. S. Vardakas, J. Abadal, C. Echave, M. M. Gúell, and C. Verikoukis, "Optimal Power Equipment Sizing and Management for Cooperative Buildings in Microgrids," IEEE Transactions on Industrial Informatics, vol. 15, no. 1, pp. 158-172, 2019.

[8] T.-H. Chang, M. Alizadeh, and A. Scaglione, "real time Power Balancing Via Decentralized Coordinated Home Energy Scheduling," IEEE Transactions on Smart Grid, vol. 4, no. 3, pp. 1490-1504, 2013.

[9] Z. Wang, C. Gu, F. Li, P. Bale, and H. Sun, "Active Demand Response Using Shared Energy Storage for Household Energy Management," IEEE Transactions on Smart Grid, vol.4, no. 4, pp. 1888-1897, 2013.

[10] W. Tushar, B. Chai, S. Huang, D. B. Smith, H. V. Poor, and Z. Yang, "Energy Storage Sharing in Smart Grid: A Modified Auction-Based Approach," IEEE Transactions on Smart Grid, vol. 7, no. 3, pp. 14621475,2016

[11] A. Jindal, M. Singh, and N. Kumar, "Consumption-Aware Data Analytical Demand Response Scheme for Peak Load Reduction in Smart Grid," IEEE Transactions on Industrial Electronics, vol. 65, no. 11, pp. 8993-9004, 2018.

[12] J. M. Guerrero, F. Blaabjerg, T. Zhelev, K. Hemmes, E. Monmasson, S Jemei, M. P. Comech, R. Granadino, J. I. Frau, "Distributed Generation: Toward a New Energy Paradigm," IEEE Industrial Electronics Magazine, vol. 4, no. 1, pp. 52-64, 2010.

[13] W. Tushar, J. A. Zhang, C. Yuen, D. B. Smith, and N. U. Hassan, "Management of Renewable Energy for an SHared Facility Controller in Smart Grid," IEEE Access, vol. 4, pp. 4269-4281, 2016.

[14] X. Chen, T. Wei, and S. Hu, "Uncertainty-Aware Household Appliance Scheduling Considering Dynamic Electricity Pricing in Smart Home," IEEE Transactions on Smart Grid, vol. 4, no. 2, pp. 932-941, 2013.

[15] S. L. Arun and M. P. Selvan, "Intelligent Residential Energy Management System for Dynamic Demand Response in Smart Buildings," IEEE Systems Journal, vol. 12, no. 2, pp. 1329-1340, 2018.

[16] N. G. Paterakis, O. Erdinc, A. G. Bakirtzis, and J. P. S. Catalao, "Optimal Household Appliances Scheduling Under Day-Ahead Pricing and LoadShaping Demand Response Strategies," IEEE Transactions on Industrial Informatics, vol. 11, no. 6, pp. 1509-1519, 2015.

[17] V.-H. Bui, A. Hussain, H.-M. Kim, and Y.-H. Im, "Optimal Energy Management of Building Microgrid Networks in Islanded Mode Considering Adjustable Power and Component Outages," Energies, vol. 11, no. 9, pp. 2351, 2018

[18] S. Althaher, P. Mancarella, and J. Mutale, "Automated Demand Response From Home Energy Management System Under Dynamic Pricing and Power and Comfort Constraints," IEEE Transactions on Smart Grid, vol. 6, no. 4, pp. 1874-1883, 2015.

[19] H. C. Jo, S. Kim, and S. K. Joo, "Smart Heating and Air Conditioning Scheduling Method Incorporating Customer Convenience for Home Energy Management System," IEEE Transactions on Consumer Electronics, vol. 59, no. 2, pp. 316-322, 2013.

[20] P. Chavali, P. Yang, and A. Nehorai, "A Distributed Algorithm of Appliance Scheduling for Home Energy Management System," IEEE Transactions on Smart Grid, vol. 5, no. 1, pp. 282-290, 2014.

[21] C. Chen, J. Wang, and S. Kishore, "A Distributed Direct Load Control Approach for Large-Scale Residential Demand Response," IEEE Transactions on Power Systems, vol. 29, no. 5, pp. 2219-2228, 2014
[22] V. Pilloni, A. Floris, A. Meloni, and L. Atzori, "Smart Home Energy Management Including Renewable Sources: A QoE-Driven Approach," IEEE Transactions on Smart Grid, vol. 9, no. 3, pp. 2006-2018, 2018.

[23] Open Government Data Platform India, "State-wise average rate of electricity for domestic and industrial consumers." [Online] Available: https://data.gov.in/catalog/state-wise-average-rate-electricity-domesticand-industrial-consumers. [Accessed: Dec. 2017]

[24] UKPower, "Fixed Rate Energy Tariffs." [Online] Available: https://www.ukpower.co.uk/home_energy/fixed-rate-tariffs. [Accessed: Dec. 2017]

[25] O. Perpinan, E. Lorenzo, and M. A. Castro, "On the Calculation of Energy Produced by a PV Grid-Connected System," Progress in Photovoltaics: Research and Applications, vol. 15, no. 3, pp. 265-274, 2007.

[26] AccuWeather, [Online] Available: https://www.accuweather.com/en/in/ patiala/190066/month/190066?monyr=12/01/2017 [Accessed: May. 2017]

[27] F. Wang, Z. Mi, S. Su, and H. Zhao, "Short-Term Solar Irradiance Forecasting Model Based on Artificial Neural Network Using Statistical Feature Parameters," Energies, vol. 5, pp. 1355-1370, 2012.

[28] G. Reikard, "Predicting solar radiation at high resolutions: A comparison of time series forecasts," Solar Energy, vol. 83, pp. 342-349, 2009.

[29] SOLARGIS. [Online] Available: http://www.solargis.info/solutions/forecast/ [Accessed: May 2018]

[30] A. Jindal, N. Kumar and J. J.P.C. Rodrigues, "A Heuristic-based Smart HVAC Energy Management Scheme for University Buildings," IEEE Transactions on Industrial Informatics, vol. 14, no. 11, pp. 5074-5086, 2018.

[31] B. Chai, A. Costa, S. D. Ahipasaoglu, C. Yuen and Z. Yang, "Optimal Meeting Scheduling in Smart Commercial Building for Energy Cost Reduction," IEEE Transactions on Smart Grid, vol. 9, no. 4, pp. 30603069, 2018.

[32] Open Energy Information, Available: http://en.openei.org/datasets/ dataset/commercial-and-residential-hourly-load-profiles-for-all-tmy3locations-in-the-united-states, [Accessed: Sept. 2018].

[33] U.S. Energy Information Administration, Annual Energy Outlook 2016, Table A4. Residential sector key indicators and consumption, Reference case: 2015. Available: http://www.eia.gov/outlooks/aeo/pdf/0383(2016).pdf. 